\title{
Lichttherapie gegen Juckreiz: UV-B nicht mit UV-A kombinieren!
}

\author{
Bei Patienten mit Psoriasis oder atopischem Ekzem bringt es für den Juckreiz offenbar nichts, \\ eine Phototherapie mit UV-B-Strahlen durch einen UV-A-Anteil zu ergänzen. Angesichts \\ möglicher kumulativer Nebenwirkungen empfehlen Schweizer Forscher, die Indikation für \\ den UV-A-Zusatz restriktiv zu stellen.
}

Die Rationale hinter einer Phototherapie bei Patienten mit chronisch-entzündlichen Hauterkrankungen besteht unter anderem darin, dass die UV-Strahlung Zellen des Immunsystems daran hindern soll, übermäßig Histamin auszuschütten. Dadurch soll der oft sehr quälende Juckreiz gelindert werden. In-vitro-Studien legen nahe, dass sowohl UV-B-Strahlen als auch UV-AStrahlen die Histaminfreisetzung aus Mastzellen inhibieren; UV-A soll darüber hinaus die Ausschüttung des Gewebshormons auch aus Basophilen eindämmen. Genau ist der Wirkmechanismus für beide Strahlungsarten jedoch nicht geklärt.

\section{Phototherapie über 16 Wochen}

Ob sich aus der Kombination kurzwelliger UV-B- mit längerwelliger UV-A-Strahlung ein klinisch relevanter Effekt auf den Juckreiz ergibt, hat ein Schweizer Team untersucht. In der randomisierten Studie absolvierten insgesamt 45 Patienten eine 16-wöchige Phototherapie, wobei die Sitzungen dreimal wöchentlich stattfanden. Bei 24 Teilnehmern bestand die Therapie lediglich aus UV-B-Strahlen mit einer Wellenlänge von $311 \mathrm{~nm}$; 21 Patienten bekamen UV-B- kombiniert mit UV-A-Licht verabreicht; die Wellenlängen lagen hier zwischen 320 und $400 \mathrm{~nm}$.

Die Teilnehmer litten überwiegend unter atopischer Dermatitis oder Psoriasis (jeweils 37\%). Als Einschlusskriterium galt ein Juckreizschweregrad von mindestens 5 auf der 10 Punkte umfassenden visuellen Analogskala (VAS). Zur Beurteilung des Therapieerfolgs wurde zusätzlich der 5-D-Itch-Score herangezogen.

$\mathrm{Zu}$ Beginn lagen die VAS-Werte in der UV-B-Gruppe bei durchschnittlich 7,2, in der Gruppe mit der UV-B-/UV-AKombination bei 7,0. Die Werte im 5-D-Itch-Score betrugen 20,9 bzw. 19,8. Beides stellte keinen nennenswerten Unterschied dar.

\section{Juckreiz in beiden Gruppen gelindert}

Nach 16 Wochen Bestrahlung hatte sich der Juckreiz in beiden Gruppen verbessert, in der UV-B-Gruppe auf einen Wert von 2,0 Punkten im VAS-Score, in der Kombi-Gruppe auf 2,5 Punkte; ein signifikanter Unterschied, ob mit oder ohne UV-A bestrahlt worden war, bestand damit nicht. Beim 5-D-Itch-Score verhielt es sich ähnlich, hier ging der Wert auf 10,5 Punkte in der UV-B- und 13,3 Punkte in der UV-B/UV-A-Gruppe zurück; auch dieser Gruppenunterschied war nicht signifikant.

Wie Julia-Tatjana Maul von der dermatologischen Abteilung der Uniklinik Zürich und ihr Team berichten, hatte sich neben dem Juckreiz auch die Krankheitsaktivität in beiden Gruppen deutlich gebessert, aber ebenfalls ohne signifikanten Unterschied zwischen den Gruppen: Von einem Ausgangswert von 12,9 in der UV-B- und 11,4 in der Kombi-Gruppe sank die Krankheitsaktivität in den 16 Behandlungswochen auf letztlich 2,2 bzw. 1,7.

Vergleichbare Effekte wurden auch beim Parameter Lebensqualität erzielt: Im DLQI (Dermatology Quality of Life Index) gingen die Werte von 11,2 bzw. 15,5 auf 5,8 bzw. 6,5 zurück. (Das Maximum von 30 Punkten steht für eine maximal eingeschränkte Lebensqualität.)

\section{Kombi-Therapie nicht überlegen}

Laut Maul und Kollegen spricht die mangelnde Überlegenheit der Kombination aus UV-A- und UV-B-Strahlung primär gegen deren Einsatz bei Patienten mit entzündlichen Hauterkrankungen und Juckreiz. Für UV-A-Strahlung sei gezeigt worden, dass sie über die Aktivierung endogener Photosensitizer DNASchäden in Hautzellen bewirken könnte; lokaler oxidativer Stress sei die Folge. Angesichts einer möglichen Kumulation von Nebenwirkungen, von Entzündungsreaktionen über eine systemische Immunsuppression bis hin zur Karzinogenese, solle grundsätzlich „der Zusatz von UV-A nicht mehr, wie bisher, empfohlen werden“.

In der Studie war es in der Kombinationsgruppe in zwei Fälle zur Exazerbation der Hauterkrankung gekommen. Dagegen waren in der rein mit UV-B bestrahlten Gruppe Nebenwirkungen ausgeblieben. Die Indikation für die Kombination UV-B plus UV-A ist nach Maul et al. im Einzelfall kritisch zu hinterfragen und sollte grundsätzlich restriktiv gestellt werden.

Dr. Elke Oberhofer

Literatur

Maul J-T et al. Impact of UVA on pruritus during UVA/B phototherapy of inflammatory skin diseases: a randomized double-blind study. J Eur Acad Dermatol Venereol. 2016; doi:10.1111/ jdv.13994 